\title{
Missing Heritability in the Tails of Quantitative Traits? A Simulation Study on the Impact of Slightly Altered True Genetic Models
}

\author{
Carolin Pütter $^{a} \quad$ Sonali Pechlivanis ${ }^{a}$ Markus M. Nöthen ${ }^{b, c}$ Karl-Heinz Jöckel ${ }^{a}$ \\ Heinz-Erich Wichmann ${ }^{d, e}$ André Scheraga \\ anstitute for Medical Informatics, Biometry and Epidemiology, University of Duisburg-Essen, Essen, \\ ${ }^{b}$ Institute of Human Genetics, and 'Department of Genomics, Life \& Brain Center, University of Bonn, Bonn, \\ ${ }^{d}$ Institute of Epidemiology, Helmholtz Center Munich, German Research Center for Environmental Health, \\ Neuherberg, and ${ }^{\mathrm{e}}$ Institute of Medical Data Management, Biometrics and Epidemiology, Ludwig Maximilian \\ University Munich, Munich, Germany
}

\section{Key Words}

Selective sampling $\cdot$ Heritability $\cdot$ Genetic association studies $\cdot$ Mixture distribution

\begin{abstract}
Objective: Genome-wide association studies have identified robust associations between single nucleotide polymorphisms and complex traits. As the proportion of phenotypic variance explained is still limited for most of the traits, larger and larger meta-analyses are being conducted to detect additional associations. Here we investigate the impact of the study design and the underlying assumption about the true genetic effect in a bimodal mixture situation on the power to detect associations. Methods: We performed simulations of quantitative phenotypes analysed by standard linear regression and dichotomized case-control data sets from the extremes of the quantitative trait analysed by standard logistic regression. Results: Using linear regression, markers with an effect in the extremes of the traits were almost undetectable, whereas analysing extremes by case-control design had superior power even for much smaller sample sizes. Two real data examples are provided to support our theoretical findings and to explore our mixture and parameter assumption. Conclusions: Our findings support the idea to
\end{abstract}

re-analyse the available meta-analysis data sets to detect new loci in the extremes. Moreover, our investigation offers an explanation for discrepant findings when analysing quantitative traits in the general population and in the extremes.

Copyright $\odot 2011$ S. Karger AG, Basel

\section{Introduction}

Genome-wide association studies (GWAS) have been very successful in detecting single nucleotide polymorphisms (SNPs) associated with complex diseases and traits or phenotypes. Despite the success, SNPs collectively account for only a small proportion of the estimated heritability of most of the complex phenotypes, this is commonly referred to as 'missing heritability' [1]. Currently, meta-analyses on permanently increasing sample sizes are performed to reveal additional associated SNPs [2-5]. One might think that with larger sample sizes, almost every influential SNP should be detectable. However, both the study design (e.g. cross-sectional design for the quantitative trait $(\mathrm{QT})$ association or case-control (CC) design) and the underlying assumption about the genetic effect (i.e. the QT distribution by genotype) have a large impact on the detectability of new associated

\section{KARGER}

Fax +4161306 1234 E-Mail karger@karger.ch www.karger.com
(C) 2011 S. Karger AG, Basel

0001-5652/11/0723-0173\$38.00/0

Accessible online at:

www.karger.com/hhe
André Scherag

Institute for Medical Informatics, Biometry and Epidemiology

University of Duisburg-Essen

DE-45122 Essen (Germany)

Tel. +49 201723 4793, E-Mail andre.scherag@uk-essen.de 
Table 1. Parameter values for the simulation studies referred to in the main text (additional results for other parameter choices are provided in the online suppl. material)

\begin{tabular}{ll}
\hline Parameters & Values \\
\hline Unimodal distribution & $\mu=0, \sigma=3$ \\
Bimodal mixture distribution & $\mu=(0,8), \sigma=(3,4), \mathrm{w}=(0.9,0.1)$ \\
Minor allele frequency (MAF) & $0.01 ; 0.05 ; 0.2 ; 0.4$ \\
Genetic effect for first normal distribution $\theta_{1}$ & $0 ; 0.05 ; 0.10 ; 0.15 ; 0.20 ; 0.25$ \\
Genetic effect for second normal distribution $\theta_{2}$ & $0 ; 0.05 ; 0.10 ; 0.15 ; 0.20 ; 0.25$ \\
\hline
\end{tabular}

SNPs. This topic has first been addressed as 'selective genotyping' of individuals with extreme phenotypes $[6,7]$ mainly for cost-efficiency reasons - initially for linkage studies [8], later for association studies [7] and most recently also for next generation sequencing [9]. General power considerations for association tests have been added $[7,10]$ and new statistical methods have also been developed [11].

Here we explore the impact of the study design and more importantly the underlying assumption about the genetic effect which has been largely ignored before for ever-growing GWAS meta-analyses. By simulating large scale cohorts and CC samples, we evaluate the power of standard association tests to detect SNP effects in the tails of a quantitative phenotype. We simulate sample sizes in the order of magnitude of the most recently published GWAS meta-analyses for standard normally distributed quantitative phenotypes and quantitative phenotypes derived from a mixture of two and more normal distributions. Two real data examples are used to underline our findings. First, we analysed the body mass index (BMI) and height quantitatively and dichotomized in a population-based cross-sectional investigation genotyped by the 'Cardio-Metabochip' ( $\mathrm{n}=4,518 ; \sim 200,000$ SNPs). Secondly, we explored our assumption of a mixed-distributed quantitative phenotype and our parameter choices for two GWAS-derived obesity SNPs in two large population-based cross-sectional investigations $(\mathrm{n}=$ 16,463). Finally, we discuss implications for future largescale GWAS meta-analyses.

\section{Materials and Methods}

\section{Simulations}

We compared linear and logistic regression analyses as standard approaches to detect a single SNP association in a GWAS by direct mapping using simulation studies. We have selected simulation parameter combinations that highlight the most interesting results, although more parameter combinations were explored (see online suppl. material, www.karger.com/doi/10.1159/000332824).

For the simulation studies, we drew $n$ genotypes under the Hardy-Weinberg equilibrium for varying minor allele frequencies (MAF; see table 1). Afterwards we drew phenotypes conditional on the genotype for two situations: (1) the standard situation of a normally distributed phenotype, and (2) the situation of a bimodal distribution resulting from the mixture of two normal distributions. Both situations are displayed in figure 1 . In the online supplementary material, extensions beyond the bimodal setting are provided.

(1) For the standard situation of a normally distributed phenotype, the expected value and variance for the phenotype conditional on the genotype were given by

$$
\begin{aligned}
& \mathrm{E}\left(\mathrm{Y}_{\mathrm{i}} \mid \mathrm{x}_{\mathrm{i}}\right)=\mu_{1}+\theta_{1} \mathrm{x}_{\mathrm{i}} \text { and } \\
& \operatorname{Var}\left(\mathrm{Y}_{\mathrm{i}} \mid \mathrm{x}_{\mathrm{i}}\right)=\sigma_{1}^{2},
\end{aligned}
$$

where $\mathrm{Y}_{\mathrm{i}}$ is the phenotype of person $\mathrm{i}, \mathrm{x}_{\mathrm{i}}$ is the given genotype. The intercept $\mu_{1}$ is the phenotype expected value for an individual without risk allele and $\theta_{1}$ is the effect of the risk allele or risk genotype. $\sigma_{1}^{2}$ is the common phenotypic variance.

(2) For the situation of a mixture of two normal distributions $\mathrm{N}_{1}\left(\mu_{1}, \sigma_{1}^{2}\right)$ and $\mathrm{N}_{2}\left(\mu_{2}, \sigma_{2}^{2}\right)$, the expected value and variance for the phenotype conditional on the genotype were given by

$$
\begin{aligned}
& E\left(Y_{i} \mid x_{i}\right)=w_{1}\left(\mu_{1}+\theta_{1} x_{i}\right)+w_{2}\left(\mu_{2}+\theta_{2} x_{i}\right) \text { and } \\
& \operatorname{Var}\left(Y_{i} \mid x_{i}\right)=\left(w_{1} \sigma_{1}^{2}+w_{2} \sigma_{2}^{2}\right)+w_{1} w_{2}\left(\left(\mu_{1}+\theta_{1} x_{i}\right)-\left(\mu_{2}+\theta_{2} x_{i}\right)\right)^{2},
\end{aligned}
$$

where $\mathrm{w}_{1}$ and $\mathrm{w}_{2}$ are weights for the mixture distribution $\left(\mathrm{w}_{1}+\right.$ $\mathrm{w}_{2}=1$ ). Similar to the situation of only one normal distribution, $\mu_{2}$ is the phenotype expected value for an individual without risk allele, $\theta_{2}$ is the effect of the risk allele or risk genotype and $\sigma_{2}^{2}$ is the common phenotypic variance for the second normal distribution, respectively.

Simulations were performed for an additive and a recessive genetic model. For the additive genetic model, we coded genotypes $\mathrm{x}_{\mathrm{i}}$ as 0,1 or 2 corresponding to the sum of the carried risk alleles of each individual. For the recessive genetic model, homozygous carriers of the risk allele were coded as $\mathrm{x}_{\mathrm{i}}=1$, whereas all other individuals were coded as $\mathrm{x}_{\mathrm{i}}=0$.

We generated 1,000 replicates for each simulation setting defined by the parameter combinations shown in table 1 that are based on empirical data from the literature [5] and our real data example 2. In each simulation setting, we investigated three ascertainment schemes: first, we simulated $\mathrm{n}=130,000$ individuals 

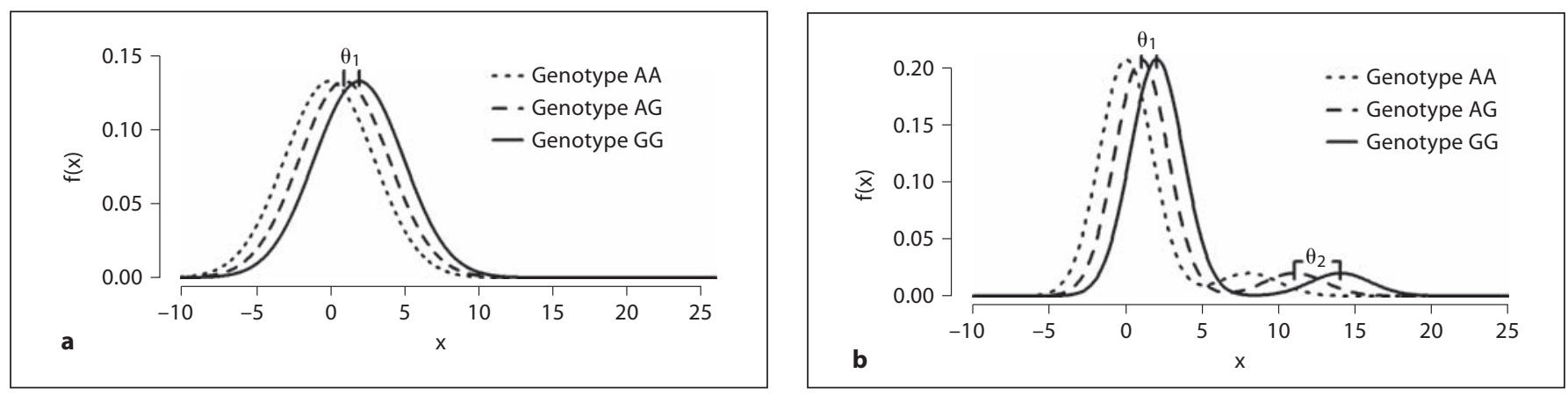

Fig. 1. Density plots by SNP genotype (arbitrarily called AA, AG and GG) for (1) the (conditionally) normal distributed phenotype (a) and (2) the phenotype of a bimodal mixture distribution (b). As examples for the investigated parameters, the figures also display the average genotypic effects $\theta_{1}$ and $\theta_{2}$ for the additive genetic model.

from the population for each replicate ('QT association') mimicking the stage I sample size of Speliotes et al. [5]. QT associations were analysed by linear regression analysis. Secondly, we selected cases and controls from the sample of $n=130,000$ individuals ('empirical CC association') or selected cases and controls from the population ('CC association'). Both CC associations were analysed by logistic regression analysis (online suppl. table 5 shows what happens if linear regression is used instead, applied to either the QT values of the individuals in the extremes or to binary (0/1)-coded outcomes). We defined cases as individuals beyond the 99th percentile of the overall phenotype distribution, while controls were defined as $\leq 50$ th percentile of the overall phenotype distribution. Note that the two CC ascertainment schemes differ: for the empirical CC association ascertainment scheme, the empirical percentile of the given $n=130,000$ individuals results in 1,300 cases and 65,000 controls (cut-off of cases: $>99$ th percentile; cut-off of controls: $\leq 50$ th percentile), whereas for the CC association scheme, the number of cases and controls is theoretically unlimited. We decided to explore 5,000 cases and 5,000 controls as a still practical setting of ongoing and future GWAS, given the medians of 700 cases and 1,200 controls for published CC studies in the GWAS catalogue [12]. In the main text, we focus on the QT association and the CC association ascertainment scheme, while the results for the empirical CC association scheme, simulation settings with altered samples sizes and altered percentile cut-offs are provided in the online supplementary material.

The comparison-wise power was defined as the proportion of replicates with $\mathrm{p}$ values for the SNP effect below the significance level $\alpha$ of $5 \times 10^{-6}$. This significance level was used by Speliotes et al. [5] for SNP selection after stage I (the SNPs followed up in the independent stage II). Note that the power for the standard situation of a normally distributed phenotype can also be evaluated analytically using tools like QUANTO 1.2.3 (http://hydra. usc.edu/gxe, [13]) or the Genetic Power Calculator [14] (available at http://pngu.mgh.harvard.edu/ purcell/gpc/).

In addition, we also ran 10,000 replicates for simulations under the null hypothesis of no genetic association for both linear and logistic regression setting and all three ascertainment schemes $\left(\alpha=5 \times 10^{-4}\right)$. In all cases we observed no evidence for an inflated empirical type I error rate (data not shown).

\section{Real Data Applications}

Example 1

To demonstrate the impact of the different ascertainment strategies, we analysed the baseline (cross-sectional)-measured BMI and height of the population-based Heinz Nixdorf Recall study (http://www.recall-studie.uni-essen.de/; details in [15]). A total of $\mathrm{n}=4,518$ individuals were genotyped by the 'CardioMetabochip' at the Department of Genomics, Life \& Brain Center. This Illumina iSELECT chip includes roughly 200,000 SNPs selected to represent genome-wide hits of large scale consortia such as the GIANT (Genetic Investigation of Anthropometric Traits) Consortium (for information on the chip design, see [16]). We followed the analysis plans of Speliotes et al. [5] for BMI and Lango Allen et al. [17] for body height and worked with the residuals which derived by adjusting for age and sex. Reflecting the proceeding in our simulations, the residuals were analyzed under a (log-) additive genetic model by linear regression for the QT association, while we used logistic regression for the empirical CC association (cut-off of cases: $>99$ th percentile; cut-off of controls: $\leq 50$ th percentile).

\section{Example 2}

To demonstrate that our assumption of mixed distributed quantitative phenotype and our chosen parameters may be reasonable, we re-analysed real data sets from two large populationbased cross-sectional investigations of European origin (total $\mathrm{n}=$ 16,463) derived from the KORA ('Cooperative Health Research in the Region of Augsburg'; http://www.helmholtz-muenchen.de/ kora/; $\mathrm{n}=12,002$ ) and the Heinz Nixdorf Recall study (see above; $\mathrm{n}=4,461)$. We focus on SNPs at two loci (FTO, MC4R) for which GWAS have first detected robust genome-wide findings. Genotyping was carried out by MALDI-TOF MS at the Helmholtz Zentrum, München, and at the Department of Genomics, Life \& Brain Center, Bonn, adapting similar quality control criteria (details in [18]). Following the analysis plan of Speliotes et al. [5], the phenotype of interest was BMI residuals which was derived by adjusting the BMI by age, age ${ }^{2}$ and sex. With the resulting BMI residuals, we fitted the genotype-stratified BMI residual distributions and visualized them in comparison to the histogram of the real data. To assess goodness-of-fit, we also perform two-sided Kolmogorov-Smirnov tests (observed data compared to best fit 


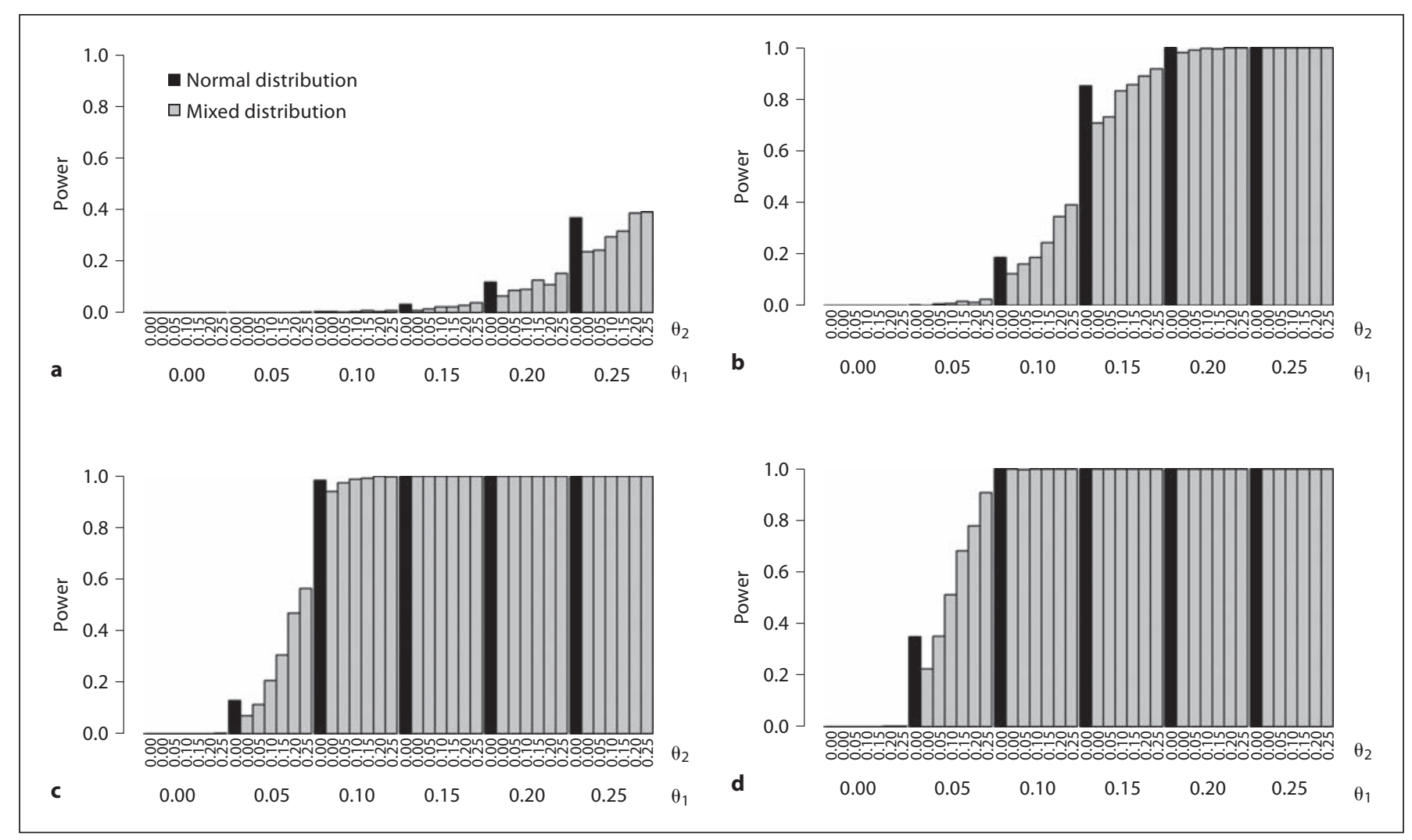

Fig. 2. QT association using an additive genetic model: the power estimates for a QT analysed by linear regression for (1) the standard situation of a (conditionally) normal distributed phenotype and (2) the situation of a bimodal mixture distribution $\left(\mathrm{n}=130,000 ; \alpha=5 \times 10^{-6} ; 1,000\right.$ replicates). MAF $=0.01(\mathbf{a}), 0.05$ (b), 0.2 (c) and $0.4($ d), respectively.

normal/mixed distribution) and derived quantile-quantile plots irrespective of genotype (see online suppl. material). The analyses were performed using the R package mixtools and norlmix.

\section{Results}

\section{Simulations}

Power Estimates for the QT Association

Figure 2 displays empirical power estimates in additive genetic models for different MAF under the standard situation of a (conditionally) normally distributed phenotype (black bars) and for the situation of a (conditionally) bimodal mixture distribution (grey bars). Even for a sample size of $\mathrm{n}=130,000\left(\alpha=5 \times 10^{-6}\right)$, the QT associations have a very limited power for MAF below 5\% under both simulated phenotype scenarios in case of small and moderate effect sizes (those below 0.15 in BMI units - see [5]). As expected, power always increased the more common the variants and the stronger the effects. Interestingly, in cases for which no ceiling effect of the estimated power was observable, power also depended on the second normal distribution of the mixture scenario. This observation was more pronounced the more frequent the investigated variant. In comparison to the standard situation of a (conditionally) normally distributed phenotype (black bars), larger power estimates of the mixture scenario were only limited to scenarios in which the effect size of the second normal distribution was stronger than the effect size for the first normal distribution.

The results of power simulation in recessive genetic models are presented in figure 3. In cases with MAF $=$ 0.01 and MAF $=0.05$, there is almost no power to detect any effect (which is why the corresponding figures have been omitted). In general, power under a recessive model was lower than power under an additive model and again the relative larger power estimates of the mixture scenario were only limited to scenarios in which the effect 

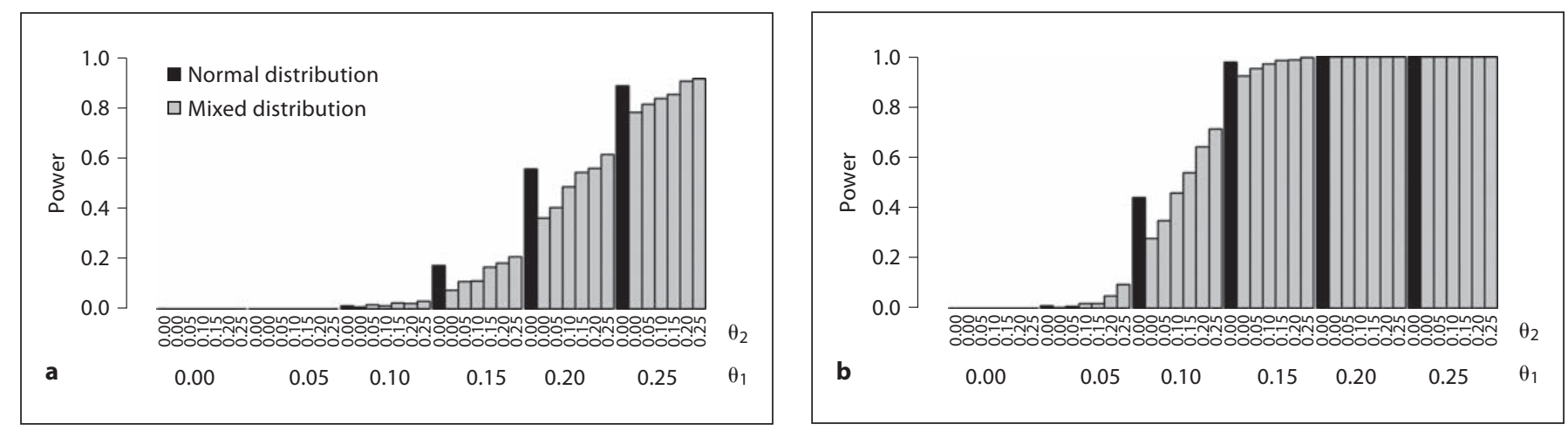

Fig. 3. QT association using a recessive genetic model: the power estimates for a QT analysed by linear regression for (1) the standard situation of a (conditionally) normal distributed phenotype and (2) the situation of a bimodal mixture distribution ( $\mathrm{n}=130,000 ; \alpha=5 \times 10^{-6} ; 1,000$ replicates). MAF $=0.2$ (a) and 0.4 (b), respectively.
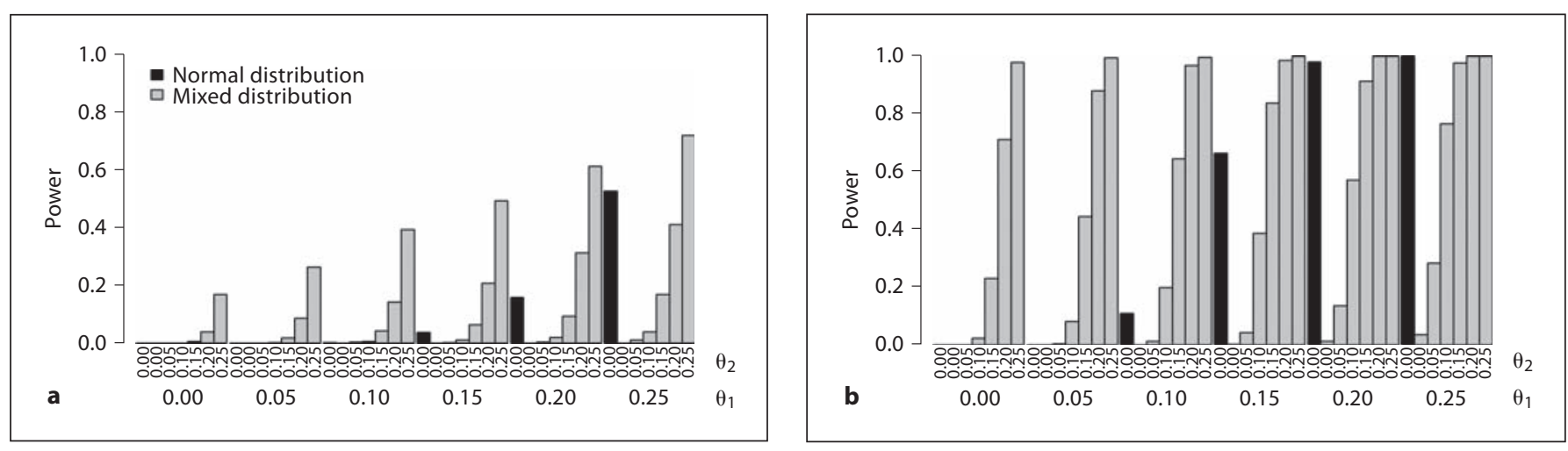

Fig. 4. CC association using an additive genetic model: the power estimates for selective genotyping of extremes from a QT for (1) the standard situation of a (conditionally) normal distributed phenotype and (2) the situation of a bimodal mixture distribution (5,000 CC pairs with cases: $>99$ th percentile, and controls: $\leq 50$ th percentile of the QT distribution; $\alpha=5 \times 10^{-6} ; 1,000$ replicates). $\mathrm{MAF}=0.05(\mathbf{a}), 0.2(\mathbf{b})$ and $0.4(\mathbf{c})$, respectively.

sizes of the second normal distribution was stronger than the effect size of the first normal distribution.

As visible in the additional simulations performed for the online supplementary material, the smaller the distance between the expected values of the underlying two or three normal distributions, the larger the resulting power estimates. With regard to variability, more variance in the extremes or shifting the weights in the bimod-

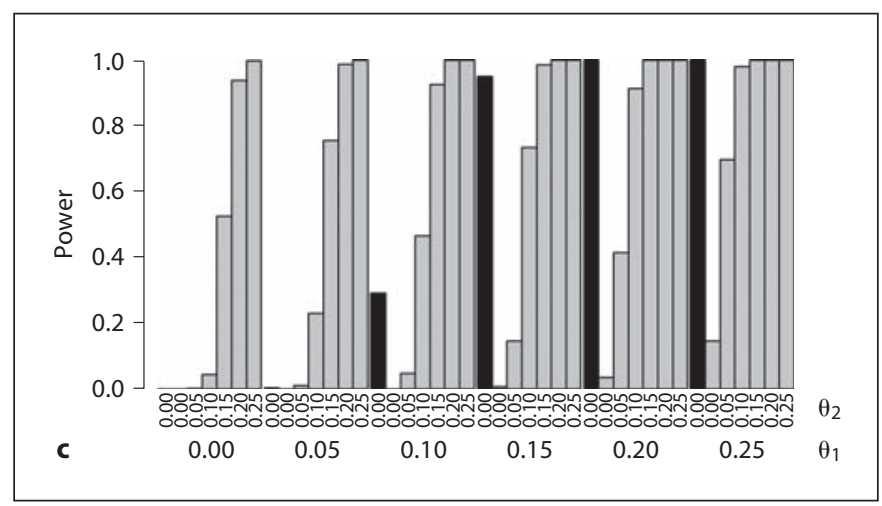

al setting from $\mathrm{w}=(0.9,0.1)$ to $\mathrm{w}=(0.8,0.2)$ had relatively little impact on the power of the QT association scheme.

Power Estimates for the CC Association

Figures 4 and 5 display empirical power estimates for the CC association scheme with 5,000 CC pairs. If $\mathrm{MAF}=0.05$ and the phenotype is (conditionally) normal distributed under an additive genetic model, power in- 


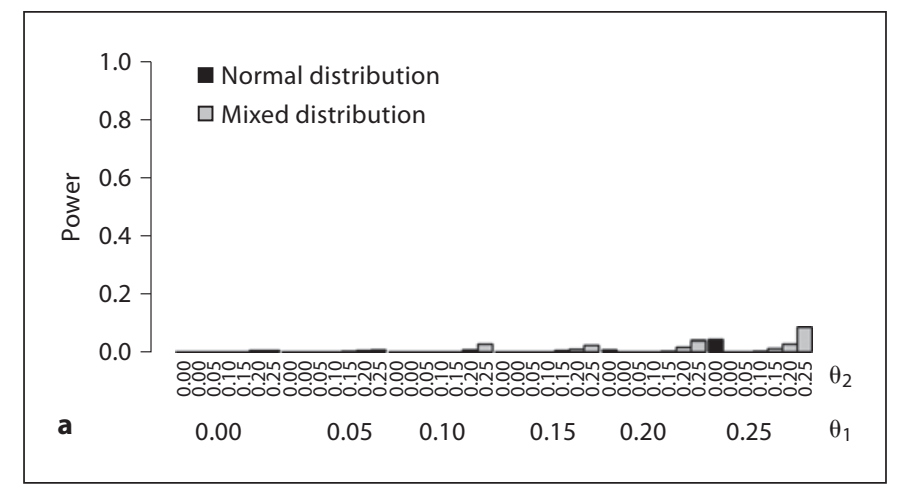

Fig. 5. CC association using a recessive genetic model: the power estimates for selective genotyping of extremes from a QT for (1) the standard situation of a (conditionally) normal distributed phenotype and (2) the situation of a bimodal mixture distribution

creases slightly in comparison to the situation of MAF = 0.01 (data not shown) and reaches a maximum of 0.53 for the largest simulated effect $\left(\theta_{1}=0.25\right)$. If the phenotype is (bimodal) mixed distributed actually, power may be further increased the stronger the effect of the second distribution $\theta_{2}$ for this maximum $\theta_{1}$. For more common variants (MAF $\geq 0.2$ ) and a conditionally normal distributed QT, CC sampling of 5,000 pairs has limited or no power to detect effects of $\theta_{1} \leq 0.15$ and this is even more extreme for a recessive genetic model (fig. 5). However, in case of mixed distributed QT with an effect $\theta_{2} \geq 0.20$ for the second normal distribution, the CC association analysis may meet a power $\geq 0.5$.

In the additional simulations performed for the online supplementary material, we observed that the more extreme the selection (also for the controls) under the CC association ascertainment scheme the larger the power estimates. For these extreme settings, varying the other parameters has little impact on power. When similarly assessing power under the empirical CC association ascertainment scheme, we observed advantages of sampling (symmetrically) from both extremes only in the prescience of effects in both tails (trimodal). Finally, under both CC association schemes (for the settings considered), the factor influencing power by far the most was the sample size of the smaller of the two (case or controls) groups.

Using linear instead of logistic regression resulted in the same pattern when compared to QT association but had a larger impact in the empirical CC association scheme (with larger power estimates for the linear regression) as compared to the balanced CC association scheme. When logistic and linear regression were applied to the same 0/1-coded outcomes, this resulted in very similar power estimates,

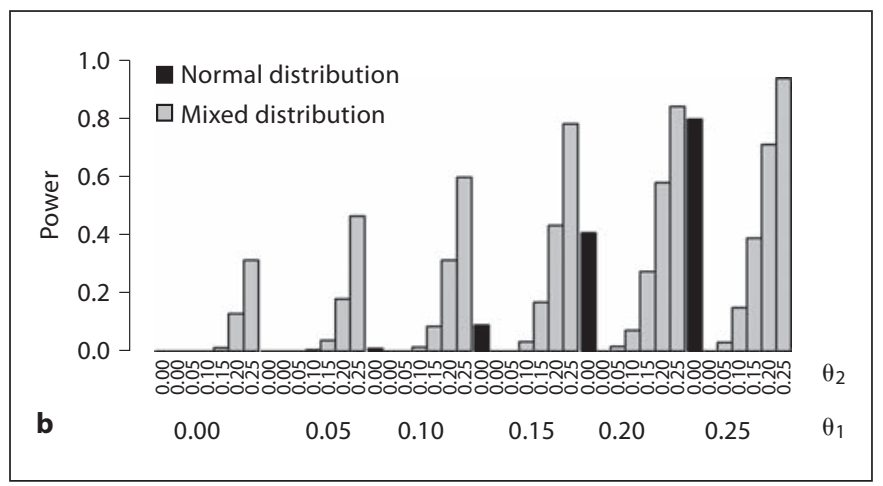

(5,000 CC pairs with cases: $>99$ th percentile, and controls: $\leq 50$ th percentile of the QT distribution; $\alpha=5 \times 10^{-6} ; 1,000$ replicates). $\mathrm{MAF}=0.2(\mathbf{a})$ and $0.4(\mathbf{b})$, respectively.

while when the original QT values were used in the linear regression, this resulted in increased power of the linear regression (as compared to the logistic regression).

\section{Real Data Applications}

Example 1

Figure 6 displays scatter-plots of the results for the QT association versus the empirical CC association (cut-off of cases: $>99$ th percentile; cut-off of controls: $\leq 50$ th percentile) for each of the roughly 200,000 SNPs of the Cardio-Metabochip. For the BMI residuals (fig. 6a), a strategy of artificially dichotomizing the data set can result in additional findings that were not detected by the QT association (at $\alpha=5 \times 10^{-6}$ ), despite the larger sample size when analysing all individuals. With a window of \pm 500 $\mathrm{kb}$ of the additional findings, the smallest $\mathrm{p}$ values for the QT association were in the range of 0.001 . In contrast, no additional findings were detected for the body height residuals (fig. 6b).

\section{Example 2}

Figure 7 displays the empirical and the fitted distributions of BMI residuals in $\mathrm{n}=16,463$ stratified by genotype (panels a and b: rs9935401 - FTO; panels c and d: rs17700144-MC4R). The better density fit was obtained for bimodal mixed distributions (panels b and d) as compared to unimodal normal distributions (panels a and c). The parameter estimates are listed in online supplementary table 6 . These estimates are comparable to the parameter choices of our simulation and may thus serve as their justification. Note that using BMI instead of BMI residuals for fitting the density reveals comparable results (data not shown). Additionally, we assessed the fit of uni- 


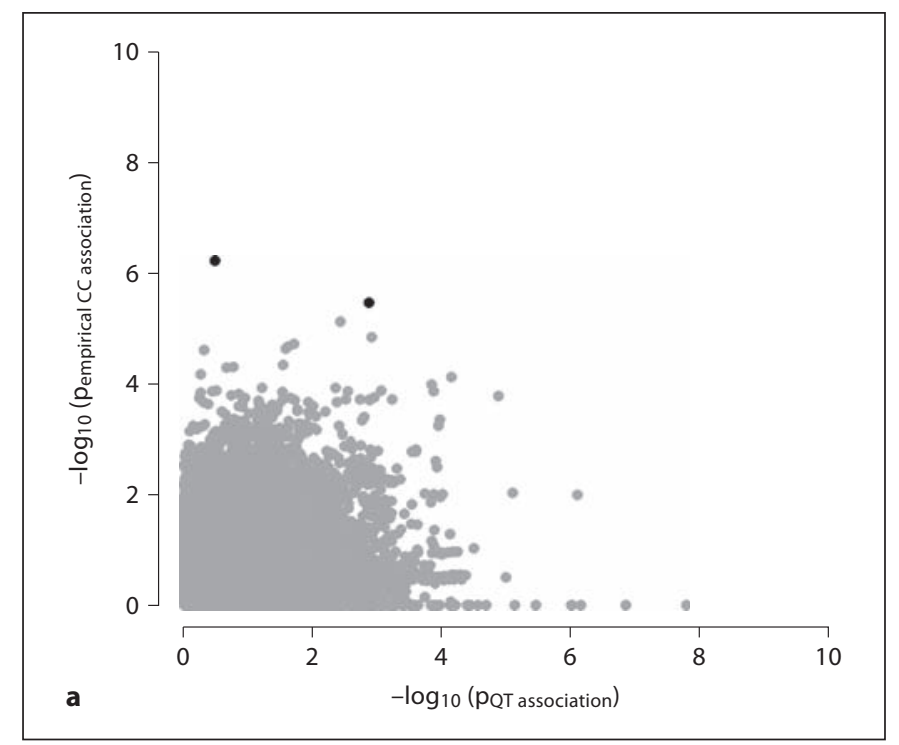

Fig.6. Example 1: scatter-plots of $-\log _{10}$ (p values) for all $\sim 200,000$ Cardio-Metabochip SNPs assessed for BMI (a) or body height residuals (b). The results for the QT association on the X-axis are plotted against the empirical CC association (cut-off of cases:

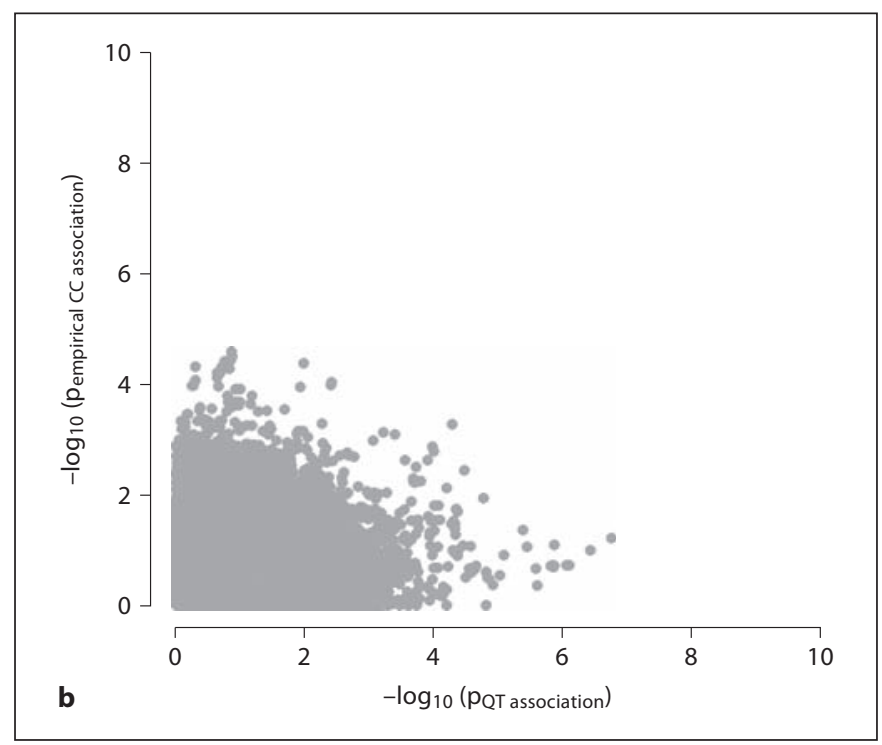

>99th percentile; cut-off of controls: $\leq 50$ th percentile) on the Yaxis. SNPs with $\mathrm{p}$ values $<5 \times 10^{-6}$ in the empirical CC association only are highlighted in black.

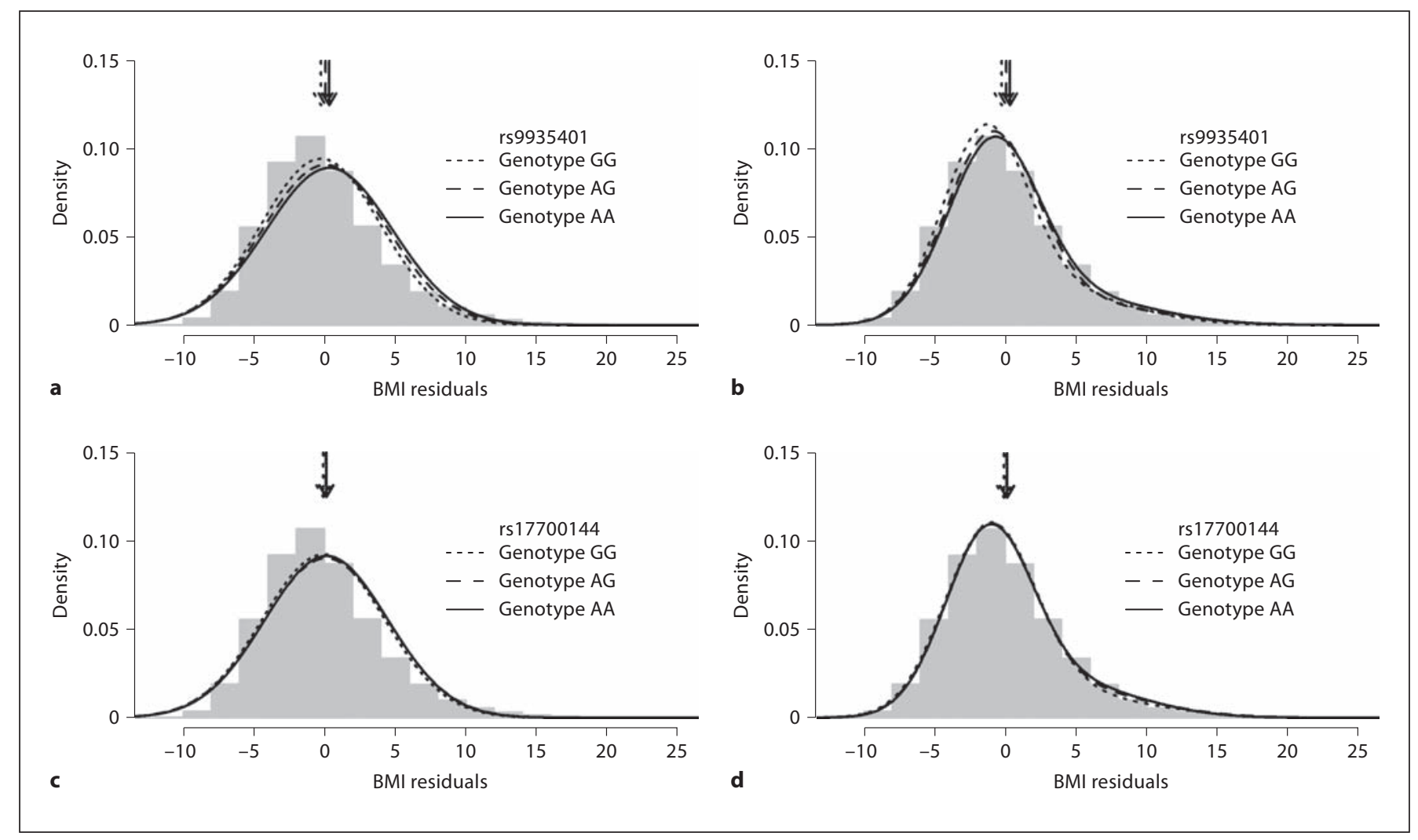

Fig. 7. Example 2: fitted BMI residuals in genotype-stratified samples (rs9935401 (FTO) and rs17700144 (MC4R)) using uni- (a, c) and bimodal, mixed distribution $(\mathbf{b}, \mathbf{d})$. The histogram in the background represents the real data; arrows indicate the estimated means. 
Table 2. QT association using an additive genetic model

\begin{tabular}{llll}
\hline MAF & $\theta_{2}$ & & \\
\cline { 2 - 4 } & 0.25 & 0.5 & 1 \\
\hline 0.4 & 0.880 & 1 & 1 \\
0.2 & 0.563 & 1 & 1 \\
0.1 & 0.163 & 0.996 & 1 \\
0.01 & 0.001 & 0.020 & 0.538 \\
0.001 & 0 & 0 & 0 \\
\hline
\end{tabular}

Values are the power estimates for a quantitative trait in a population-based study analysed by linear regression and with a sample size of $\mathrm{n}=1,000,000$ when an effect is only present in the extremes $\left(\theta_{1}=0\right)$.

and multimodal models to BMI residuals using quantilequantile plots and Kolmogorov-Smirnov goodness-of-fit tests (see online suppl. material). These comparisons indicated a better fit (particularly in the tails) in case of the mixture settings.

\section{Discussion}

There is an ongoing trend to perform GWAS metaanalyses at an ever growing size in order to detect most of the variants that contribute to the genetic architecture of a complex phenotype. While for the largest part our simulation results support this view, we also found support for the idea of exploring samples selected from the extremes of a phenotype to detect new and other genetic variants.

We demonstrated that if we alter the assumption of a (conditionally) normally distributed trait to a slightly more complicated mixture setting, investigators may be able to detect new genetic variants using selective genotyping at the extremes of the quantitative phenotype (even for much smaller sample sizes). Moreover as displayed in table 2, further increasing the sample size of population-based samples under the QT association ascertainment scheme to e.g. $\mathrm{n}=10^{6}$ is of little help in case the genetic effect is largely driven by an effect in the extremes. This is simply due to the fact that the majority of individuals influence the QT association linear regression result, while the few individuals in the extremes have little impact. This observation has at least three implications. First, it may contribute to an understanding why some of the recent GWAS meta-analyses adapting either a population-based QT association linear regression view (e.g. [5]) as compared to an extremes (CC) view (e.g. [18]) may come up with slightly different result sets in addition to the possibility of a false-positive finding. Second, both our simulations and the real data example 1 support the idea that running a meta-analysis selecting cases and controls from the tails of a phenotype may be worthwhile to detect new associated variants. Third, as demonstrated in the real data example 2, estimating parameters from a mixture distributions setting and comparing them to the standard analysis may also add to our understanding of the genetic architecture of some complex traits.

Besides these conclusions, particularly our simulation study also has some limitations. We generated data sets of QT loci and SNP genotype data that were derived from empirical estimates (see example 2) and were thought to mimic the ongoing search of associated SNPs $(n=130,000$ at $\alpha=5 \times 10^{-6}$ or 5,000 CC pairs also at $\alpha=5 \times 10^{-6}$ ). Given the very different sample sizes, power estimates are not comparable between the ascertainment schemes as has already been discussed intensively by others (e.g. [10, 19]). Please note, that the sample size of 5,000 cases selected using a $\geq 99$ th percentile phenotype cut-off may require a cross-sectional study size of $n=500,000$ under the empirical CC association scheme. Furthermore our findings do not result from contrasting linear and logistic regression, as analysing the CC ascertainment schemes by linear regression resulted in a very similar observation if $0 / 1$-coded outcomes were considered. Our choice of $\alpha=5 \times 10^{-6}$ may be regarded as a too liberal significance level. However, given the monotone relationship between power and significance level, the power estimates obtained here will likely be an overoptimistic view. Furthermore the choice of a bimodal mixture of two normal distributions may seem relatively arbitrary. We presented the real data example 2 for BMI to show the superior fit of the mixture densities which may of course only be due to the larger flexibility of a mixture density situation. Despite this real data example, the bimodal mixture situation remains a model of a slightly more complicated genetic architecture of a complex trait. This model might match the empirical findings on both common and extreme forms of complex traits - see [20] for examples related to type 2 diabetes and obesity. Extending the mixture setting to more than two normal distributions requires setting additional simulation parameters. Exploring some trimodal mixture settings in general showed that effects in both extremes/tails may be detected best by selecting extreme cases and extreme controls from the population. Finally, one may question when power differences are expected, given that larger power estimates were often limited to mixture settings in which the effect 
sizes of the second normal distribution were larger than the first normal distribution. This question can only be accessed empirically - most likely the answer will be different for different phenotypes (see also our example 1 contrasting BMI and height).

In sum, we have supported the idea that selective sampling and selective analysis of samples may contribute to the detection of new genetic variants of complex traits in addition to the ones detectable by large scale populationbased analyses. The future will show if the model propagated to support this claim turns out to be useful for detecting novel genes related to complex traits.

\section{Acknowledgements}

We thank all participants of this study. We also thank S. Moebus and R. Erbel for the Heinz Nixdorf Recall Study, T. Illig for the KORA study and D. Schadendorf and T.W. Mühleisen for their support of the Cardio-Metabochip project.
IRB Approval

The study, including the protocols for subject recruitment and assessment and the informed consent for participants, was reviewed and approved by all local IRB boards and was carried out in accordance with the Declaration of Helsinki.

\section{Funding}

This work was supported by grants from the German Ministry of Education and Research (01KU0903; NGFN"plus: 01GS0820). We thank the Heinz Nixdorf Foundation, Germany, for the generous support of the Heinz Nixdorf Recall Study. The KORA Augsburg studies were financed by the Helmholtz Zentrum München - Research Center for Environment and Health, Neuherberg, Germany, and supported by grants from the Bundesministerium für Bildung und Forschung and the Munich Center of Health Sciences (MC Health) as part of the Ludwig Maximilian University innovative.

The funders had no role in the study design, data collection and analysis, decision to publish, or preparation of the manuscript.

\section{References}

1 Manolio TA, Collins FS, Cox NJ, Goldstein DB, Hindorff LA, et al: Finding the missing heritability of complex diseases. Nature 2009;461:747-753.

-2 Elks CE, Perry JR, Sulem P, Chasman DI, Franceschini N, et al: Thirty new loci for age at menarche identified by a meta-analysis of genome-wide association studies. Nat Genet 2010;42:1077-1085.

-3 Franke A, McGovern DP, Barrett JC, Wang $\mathrm{K}$, Radford-Smith GL, et al: Genome-wide meta-analysis increases to 71 the number of confirmed Crohn's disease susceptibility loci. Nat Genet 2010;42:1118-1125.

-4 Schunkert H, Konig IR, Kathiresan S, Reilly MP, Assimes TL, et al: Large-scale association analysis identifies 13 new susceptibility loci for coronary artery disease. Nat Genet 2011;43:333-338.

- 5 Speliotes EK, Willer CJ, Berndt SI, Monda KL, Thorleifsson G, et al: Association analyses of 249,796 individuals reveal 18 new loci associated with body mass index. Nat Genet 2010;42:937-948.

-6 Lander ES, Botstein D: Mapping mendelian factors underlying quantitative traits using RFLP linkage maps. Genetics 1989;121:185199.

7 Van Gestel S, Houwing-Duistermaat JJ, Adolfsson R, van Duijn CM, Van Broeckhoven C: Power of selective genotyping in genetic association analyses of quantitative traits. Behav Genet 2000;30:141-146.

8 Purcell S, Cherny SS, Hewitt JK, Sham PC: Optimal sibship selection for genotyping in quantitative trait locus linkage analysis. Hum Hered 2001;52:1-13.

9 Guey LT, Kravic J, Melander O, Burtt NP, Laramie JM, Lyssenko V, Jonsson A, Lindholm E, Tuomi T, Isomaa B, Nilsson $\mathrm{P}$, Almgren P, Kathiresan S, Groop L, Seymour $A B$, Altshuler D, Voight BF: Power in the phenotypic extremes: a simulation study of power in discovery and replication of rare variants. Genet Epidemiol 2011, DOI: 10.1002/gepi.20572.

10 Darvasi A, Soller M: Selective genotyping for determination of linkage between a marker locus and a quantitative trait locus. Theor Appl Genet 1992;85:353-359.

11 Huang BE, Lin DY: Efficient association mapping of quantitative trait loci with selective genotyping. Am J Hum Genet 2007;80: 567-576.

12 Hindorff LA, Junkins HA, Hall PN, Mehta JP, Manolio TA: A Catalog of Published Genome-Wide Association Studies. Available at: www genome gov/gwastudies (accessed 07/06/2011)

13 Gauderman WJ, Morrison JM: QUANTO 1.1: A computer program for power and sample size calculations for genetic-epidemiology studies. http://hydra.usc.edu/gxe, 2006.

14 Purcell S, Cherny SS, Sham PC: Genetic power calculator: design of linkage and association genetic mapping studies of complex traits. Bioinformatics 2003;19:149-150.

15 Schmermund A, Mohlenkamp S, Stang A, Gronemeyer D, Seibel R, Hirche H, Mann K, Siffert W, Lauterbach K, Siegrist J, Jockel KH,
Erbel R: Assessment of clinically silent atherosclerotic disease and established and novel risk factors for predicting myocardial infarction and cardiac death in healthy middle-aged subjects: rationale and design of the Heinz Nixdorf RECALL Study. Risk Factors, Evaluation of Coronary Calcium and Lifestyle. Am Heart J 2002;144:212-218.

16 Pechlivanis S, Scherag A, Pütter C, Moebus $S$ : Metabochip - a next generation genotyping platform; in Hemmelmann C, Ahnert $\mathrm{P}$, Foraita R, Großhennig A, Scherag A, Biebler K-E (eds): Biometrische Aspekte der Genomanalyse IV, Next Generation Sequencing Data Analysis. Aachen, Shaker Verlag, 2011, pp 47-54.

17 Lango Allen H, Estrada K, Lettre G, Berndt SI, Weedon MN, et al: Hundreds of variants clustered in genomic loci and biological pathways affect human height. Nature 2010; 467:832-838.

-18 Scherag A, Dina C, Hinney A, Vatin V, Scherag S, et al: Two new loci for body-weight regulation identified in a joint analysis of genome-wide association studies for early-onset extreme obesity in French and German study groups. PLoS Genet 2010;6:e1000916.

19 Yang J, Wray NR, Visscher PM: Comparing apples and oranges: equating the power of case-control and quantitative trait association studies. Genet Epidemiol 2010;34:254257.

20 McCarthy MI: Genomics, type 2 diabetes, and obesity. N Engl J Med 2010;363:23392350 